\title{
Perceptions of the nocturnal public space and technological innovations - citizen evaluation of a smart public lighting installation in Nantes
}

\author{
Nicolas Houel1,*, Dany $\mathrm{Joly}^{2}$, Laurent Lescop ${ }^{3}$ \\ ${ }^{1}$ AAU Laboratory, CRENAU team, ENSA Nantes 6 quai François Mitterrand 44000 Nantes, France \\ ${ }^{2}$ EPICE department, Nantes Métropole, 5 rue de Saverne 44000 Nantes, \\ ${ }^{3}$ AAU Laboratory, CRENAU team, ENSA Nantes 6 quai François Mitterrand 44000 Nantes, France
}

\begin{abstract}
The recent introduction of digital tools in public lighting represents a milestone in the evolution of street lighting. Simultaneously to this innovation phase, urban lighting is currently being studied in terms of energy and environmental sobriety, to reduce its financial and ecological impact. This study presents the observations and evaluations carried out by nocturnal urban dwellers of an experimental public lighting system, set up in real conditions. The evaluations question the relevance of sensors integrated to the lighting material in real time via a series of survey managed autonomously by residents of the experimental environment.
\end{abstract}

\section{Introduction}

The leading position of public lighting in urban spaces, determined by progressive and innovative approaches that are still topical [1][2] has since long been supported by initiatives dealing with the safety of people and property, traffic, and the balance between the electricity production and consumption in France [3]. Beyond deeply contrasted nocturnal landscapes between rural and urban environments [4], this attention has consolidated and generated some beliefs in terms of nocturnal urban practices and related artificial lighting. Among those, while the connection between lighting and feelings of safety is definitely rooted [5][6], and the association between visual comfort and the amount of light is present in several urban lighting projects, new lighting and communication technologies, i.e. LED and wireless networks, introduce a new type of beliefs: one in technology for communities and citizens. With technological development comes a wider range of choices, criteria, accessories and ambiances than before, i.e. high-pressure sodium lights and metal halide lamps. In that sense, how can communities, with a public lighting stock in constant renovation, face the choices and arguments of the manufacturers? More specifically, at a time when lights can provide additional services, adjust their lighting levels in real time and change their light temperature to fit the needs, the following question arises: how to illuminate? Led by the leitmotiv by the Association Française de l'Éclairage, some communities are currently exploring the necessary tools to determine the needs and uses for their nocturnal territories, as to provide

\footnotetext{
* Corresponding author: nicolas.houel@crenau.archi.fr
} 
adapted lighting configurations. Thereon, Nantes Métropole, with its advisory experiences (Major debates on energy transition ${ }^{2}$, permanent conference on the Loire ${ }^{3}$, and, at the municipal level, the Conseil Nantais de la Nuit ${ }^{4}$ ), is interested in the ways to encourage citizen involvement in the development of their Public Lighting Equipment Scheme (Schéma de Cohérence d'Aménagement Lumière - SCAL), designed to support the public lighting renovation in the metropolis' 24 municipalities. In that respect, the perception studies on artificial lighting carried out in the 2000s in France [7][8][9], and the current initiatives on nocturnal wandering to observe public space at night (Sara Castagné ${ }^{5}$ and Isabelle Corten ${ }^{6}$, lighting designers in France, and Léni Schendinger ${ }^{7}$ in the United States) strengthen the baseline for qualitative studies for the observation of the nocturnal public space, and allow to explore tools to collect physical (urban fabric configurations, lamps) and sensory (perceptions of the nocturnal public space, of the lighting) indicators. The citizens are thus invested and contribute to the knowledge input that is wanted nowadays. As part of SCAL, the first stage - evaluation of tools and methods to collect indicators - was carried out on the Nantes Métropole territory in 2018. The chosen protocol questioned several beliefs supported by the citizens and the communities, namely: Are the citizens' views relevant in terms of environmental considerations about artificial lighting? Is technological innovation, in particular the solution of embarked sensors, of interest for the communities? Are lighting level and comfort (vision, feeling of safety) connected? Lastly, the experiment led to the evaluation of acceptability regarding a dynamic lighting system, in which the light intensity changed depending on the movements detected in the street. For this study, the experiment relied on the implementation of ten experimental lamps equipped with various sensors, including a motion sensor, set up in Nantes Métropole's public space. Configurable in terms of intensity level and dimming speed, they were assessed by citizens twice. Carried out independently, the citizen evaluations led to the creation of two grading systems: one was physical (paper) and the other digital (online survey). Based on the results, it aims to question the relevance of a lighting system with varying intensity and its acceptability according to different parameters (intensity level, dimming speed). The first results of the investigation, taking place at the level of a street, aim to contribute to the elaboration of a collection tool for physical and sensory indicators on a bigger scale, to build a quantitative and qualitative database about nocturnal public space and its perceptions. Therefore, the study questions the current intuitions and beliefs regarding public space at night, lighting material and their evolutions. Indeed, if the first results appear to highlight contradictions with the established beliefs, the limited number of the panel calls for reproducing this evaluation on a bigger scale, in order to confront the results and assess the state of beliefs and potential objections in a quantitative way. Lastly, reproducing this system on a bigger scale would allow to participate in the implementation of a form of education about lighting sobriety for the communities and their users, thus allowing to talk about light extinction and dimming policies in public space, or the concept of dark scheme in a collective way, to contribute to the current research on fair lighting.

\footnotetext{
${ }^{2}$ https://www.nantestransitionenergetique.fr/

${ }^{3}$ https://www.nanteslaloireetnous.fr/

${ }^{4}$ https://www.nantes.fr/conseil-nuit

${ }^{5} \mathrm{https}: / / \mathrm{www}$. lightzoomlumiere.fr/interview/sara-castagne-mettre-en-lumiere-en-coconception/

${ }^{6} \mathrm{http}: / /$ www.radiance35.eu/fr/agence

${ }^{7} \mathrm{http}: / /$ www.nightseeing.net/
} 


\section{Spatial and material environment}

The study took place in the rue La Noue Bras de Fer in Nantes, on a 250m linear segment, located between the Boulevard Léon Bureau/rue La Noue Bras de Fer junction and the Manny building. Over this area, ten $150 \mathrm{~W}$ high-pressure sodium lamps were substituted with ten LED dimmable lamps, which are $15 \mathrm{~W}$ in standby mode and $45 \mathrm{~W}$ in activity. The location for the experiment was chosen according to the following indicators: presence of a bidirectional traffic lane, of pedestrian accesses on each side of the road, of parking spots on one side, commuting traffic at the beginning and end of the day, presence of vegetation (medium-height trees), diversity in the uses of the site (residential, professional, commercial, vehicular and soft traffic). The road and the pavements are lined with medium-height contemporary buildings with different façade treatments, two junctions are identified, as well as vegetal space. Lastly, one of the determining elements was the presence of a Linky metre connected to the control cabinet for the selected light points. It allows to quantify the expected energy savings. The ten smart lamps that were deployed are the results of the Interactive Data Light project ${ }^{8}$, award-winner of the 2017 Challenge pour l'Accélération Digitale de l'Ouest. Designed by a consortium of Nantes-based companies, they are equipped with the following: air quality sensor, decibel level sensor, temperature and hygrometry sensor, energy consumption, motion sensor. Each lamp independently captures the data related to the sensors, then sends it via a wireless communication protocol to the master-lamp $\left(\mathrm{n}^{\circ} 1\right)$, located at the beginning of the street and equipped with a $3 \mathrm{G}$ communication system to send it to a server. The communication protocol enables a precise activation of the lamps: when a $n$ lamp senses movement, it changes its light intensity (10\% of the flux to $30 \%)$ and communicates the same order to its $n-1$ and $n+1$. Therefore, regardless of the direction of the detected object, its path is illuminated. On each lighting column, a kakemono banner is set up to indicate the experimentation object and invite the pedestrians to fill out an online questionnaire. It aims to collect the sensory indicators from the users of the experimentation space, in terms of their perceptions of dimmable lighting.

\section{First study: voluntary and autonomous assessment of lighting}

To this day, lighting with varying intensity is little developed in public space. While its values regarding energy savings and reduction of light pollution can be verified - during the evaluation - , knowledge of the users' sensory perceptions of the nocturnal public space is currently researched [10][11], in particular regarding lighting levels, illuminated areas and related feeling of safety. The observation of the current evaluation protocols for public lighting demonstrates that their spatial and temporal conditions are determined for the assessment. Therefore, the site or itinerary and the beginning and end of the evaluation are established a priori, and often realized in a controlled environment (street without cars, enclosed university campus, etc.). Moreover, a panel of evaluators is constituted in advance, and they sometimes receive financial compensation. For the first study, the environment to assess is not adapted to the evaluation: it maintains its usual traffic flows and uses. Additionally, no panel of evaluators is determined in advance. These two elements allow to consider the collection of sensory indicators in real conditions, and to observe a pedestrian's proper initiative, to be completed with the online questionnaire.

The ten-question survey aims to identify the evaluation conditions (time when they crossed the street, means of transport), profile of the evaluator (age, gender) and the perceptions regarding lighting (identification of the variation in intensity, sensation of comfort, sensation of discomfort, feeling of safety). Fifteen evaluators participated, on their

\footnotetext{
${ }^{8}$ https://www.nicolashouel.fr/?p=493
} 
own accord and autonomously, in the survey. Parity is almost total, with seven women and eight men. Among them, two are between 18 and 24 years old, ten between 25 and 45 years old, one is between 46 and 59 years old and one is over 60 years old.

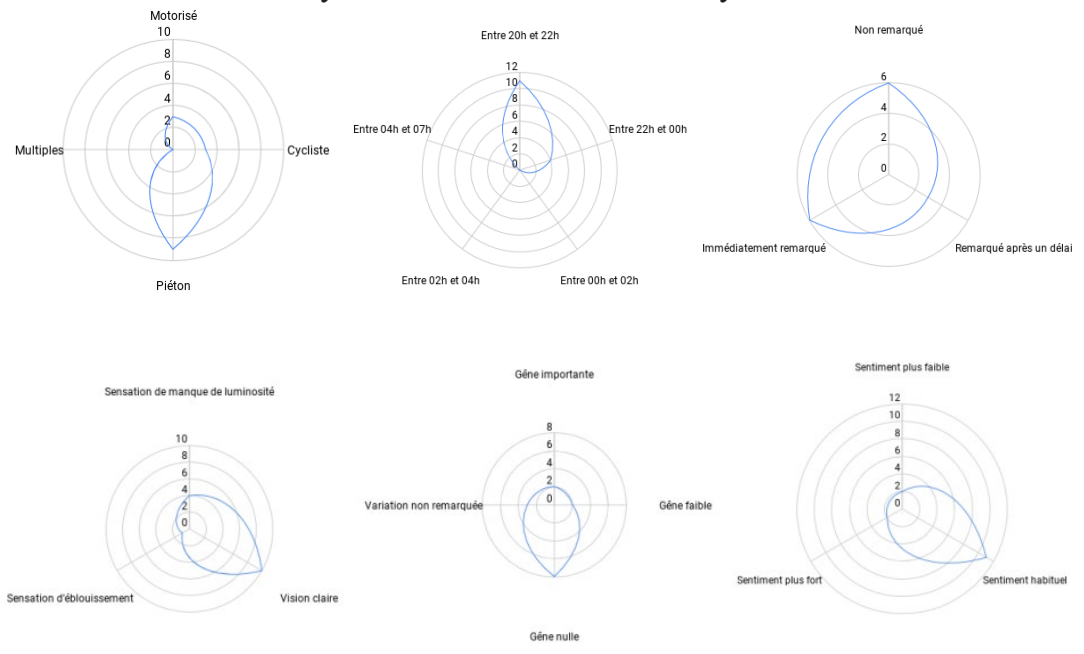

Fig. 1. Radar profiles of the collected data $-1^{\text {st }}$ line (a), (b), (c) $; 2^{\text {nd }}$ line (d), (e), (f).

The collected data allows us to see that most evaluators are pedestrians (Figure 1a, val. 9), and cross the street between $8 \mathrm{pm}$ and $10 \mathrm{pm}$ (Figure 1b, val. 11). The variation of intensity by motion sensor is balanced between a complete absence of notification (Figure 1c, 6), and a direct notification of the variation (Figure 1c, val. 6). The luminosity proposed in active mode (30\% of intensity, around $18 \mathrm{lux}$ ) is largely considered as correct (Figure 1d, val. 10). Increasing the light level, from 10\% (around 8 lux) to 30\% (around 18 lux) causes a slight discomfort in $13 \%$ of the cases. The same number corresponds to strong discomfort. However, the discomfort is null in $73 \%$ of the evaluations (Figure 1e). Lastly, the feeling of safety is assessed as usual in $73 \%$ of the cases (Figure 1f, val. 11). Two evaluators consider that they feel less safe, and two people think that they are safer. These first observations allow us to hypothesise that a public lighting system with variation of intensity, respectful of the normative values of lighting at high level and inferior to the same criteria in reduced flux, could be considered as acceptable by the pedestrians, at least in the temporal and spatial conditions of the experiment site. Set up between the end of April 2018 and the middle of September 2018, the promotion of the evaluation of this experimental system was disseminated in the media and on the social media pages of some of the project's institutional partners. The relatively small number of evaluators lastly questions the importance given by individuals to public lighting. Several hypotheses can explain this phenomenon: poor legibility of the communication, set up above eye level, reduced typographical characters; the length and complexity of the URL to access the questionnaire (http://www.jeparticipe.idlsmartcity.com/), and the evaluation period, given that night falls later during the spring and summer. The number of pedestrians at this time might have been too small to result in a quantitative study. One last element remains to be studied: the comments, left to the discretion of the evaluators. Out of the fifteen participants, four of them have left a comment: 
1/ Very useful against light and atmospheric pollution.

2/ Try this on the Pont de Chèvre [Editor's note: Sèvre] that has not been illuminated for years. Unacceptable in rainy times in the winter, we can't see anything, not even the exits. Spend tax money efficiently.

$3 /$ This is a nice project, to be multiplied in other streets in Nantes and around.

4/ Light out of service on May ${ }^{\text {st }}$, at $9.45 \mathrm{pm}$.

These comments seem to be coherent with the sensory evaluations collected before the questionnaire. Moreover, we can note an awareness to environmental concerns (1), a possible way of identifying breakdowns (4), and the possibility to think about the design of public lighting in collaboration with the citizens, comments (2) and (3) stating the desire to multiply the system and the identification of a potential intervention site.

\section{Study $n^{\circ} 2$ : evaluation of lighting by residents}

Integrated within a broader research project about education on lighting sobriety, the second study aims to observe the ability of a panel of evaluators to contribute to the establishment of a knowledge base on nocturnal uses of a territory. This study also aims to evaluate the awareness level regarding current considerations on artificial lighting (energy savings, night sky pollution, damage to the fauna and flora). In that sense, an evaluation protocol on nocturnal perceptions in the rue La Noue Bras de Fer was established as follows: four successive weeks for the evaluation with six questionnaires, three meetings between evaluators and project leaders (presentation, collaboration, conclusion). During these four weeks, three changes in the light intensity of the lamps (Table 1) were implemented, then three changes in the dimming parameters (Table 1).

Table 1. Configuration of the parameters for the variation in intensity and dimming, by stage.

\begin{tabular}{|c|c|c|}
\hline Étape & Intensité (veille > activité, en \% d'intensité) & vitesse (veille > activité > veille, en ms) \\
\hline 1 & $10>30$ & $500>2000>500$ \\
\hline 2 & $10>30$ & $500>2000>500$ \\
\hline 3 & $10>20$ & $500>2000>500$ \\
\hline 4 & $10>20$ & $100>2000>500$ \\
\hline 5 & $10>20$ & $500>1000>500$ \\
\hline 6 & $10>20$ & $500>3000>500$ \\
\hline
\end{tabular}

Following the first study, the evaluations were carried out autonomously by the residents. Only a reminder via email was sent the day before a change in the lighting parameters. No information was given about the modifications themselves, as the aim was to observe the general perceptions of the street ambiance, independent from the sole observation of artificial lighting. In order to help the procedure, the evaluation questionnaire came in two ways: a paper and a digital format. The tester's log book included, for each step, a transcript of the physical conditions of the evaluation (time where they crossed the street, weather conditions, site traffic, traffic direction of the evaluator) and five questions according to the protocol established by Boomsma \& Steg (2014). Within the study, the questions suggested by Boomsma \& Steg, on the feeling of insecurity, were rewritten to focus on the feeling of 
tranquillity. To this end, we used IATE $^{9}$ and the $\mathrm{CNRTL}^{10}$ thesaurus to study the potential positivity in the perceptions, with the hypothesis that a series of affirmative questions could perhaps guide the evaluator's perceptions. Figure 4 presents the questions that were submitted to the participants. Each one was subject to a notation system, like Lickert's scale, from 1 (complete disagreement) to 5 (complete agreement).
A. I found the ambiance pleasant.
B. Ifelt safe.
C. If I didn't have to go through this street for the evaluation today, I would have liked to go through another street.
D. I changed my walking pace in the street.
E. I would have liked to see more people in the street.

Fig. 2. series of questions asked to the evaluators, for each stage of the evaluation.

The data collected during the second study seems to present positive results in terms of modification of public lighting parameters. As shown in Figure 3, the perceptions of a nocturnal public space in real conditions (automobile traffic, construction works in the street, varying weather conditions, varying crowd, potential glare by car lights, etc.) seem little affected by the transformations in artificial lighting. More specifically, only the dimming speed parameters, in particular during the transition between standby and active mode are perceived as blinding when they are too quick $(100 \mathrm{~ms})$. Otherwise, the decrease in lighting level is not perceived, and thus does not seem to affect the feeling of comfort and safety.

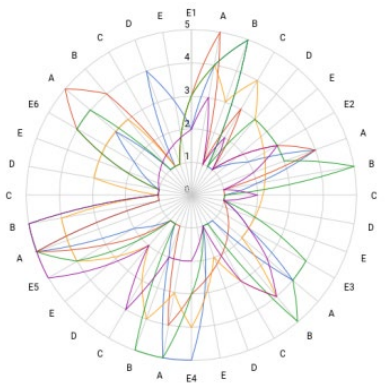

Fig. 3. Overview of the evaluations. The Ens represent the evaluation stages, the A to E letters represent the questions (cf. Fig. 2) for each stage.

The decision to select a panel of residents from the experiment site aimed to facilitate the autonomous evaluation over a certain period of time and the implementation of meetings. The first one was a presentation of the evaluation project on nocturnal perceptions of the rue La Noue Bras de Fer, and the collection tools of these indicators. The second meeting was a

\footnotetext{
${ }^{9} \mathrm{https}: / /$ iate.europa.eu/home

${ }^{10} \mathrm{http}: / /$ www.cnrtl.fr/
} 
restitution stage by the evaluators (three filled-out questionnaires per person) and allowed to test an evaluation tool on current public lighting, i.e. an interactive digital map that displays colour gradients regarding the power of the 95,000 lamps in Nantes Métropole. Projected horizontally on a glass table, the map allowed to learn about Nantes Métropole's entire public lighting stock, as well as zoom in and out of specific areas. With this tool, the residents were able to give a series of sensory indicators on nocturnal perceptions of spaces they are used to visit. The first evaluator told us that a road along the Loire could benefit from varying lighting to reduce the light flux outside the commuting times, and thus sustaining biodiversity. Another evaluator then shared his difficulties to ride his bike on a specific route, as the path was not properly illuminated. Lastly, a third evaluator mentioned that she did not feel safe on a section of her everyday walk, in an area where the lighting was lacking, in her opinion. While this last contribution suggests the current importance of lighting in the feeling of safety [5], the first two comments on artificial lighting, i.e. nuisance for the ecosystems and the need to accompany the rise of urban soft mobilities with lighting equipment that is adapted at nightfall. Moreover, this last point is one of the major entries in the revision of the Nantes Métropole's Public Lighting Equipment Scheme and is in continuation with the renewed use of soft mobilities (cycles, gyropods, scooters, etc.) that recently appeared in the city. The simultaneity of the SCAL development and of these new transportation modes could be studied in more details, for a way to illuminate soft modes of transport and understand nocturnal landscapes with darkness [12][13][14].

\section{Conclusion}

The two studies aimed to explore three current questions: Are the citizens' views relevant in terms of environmental considerations about artificial lighting? Is technological innovation, in particular the solution of mobile sensors, of interest for the communities? Are lighting level and comfort (vision, feeling of safety) connected? For each question, the results collected in the studies are encouraging. With their comments during the second study, and in particular during the manipulation of the cartographic tool, the evaluators seemed aware of environmental questions regarding artificial lighting, and willing to reduce lighting to sustain the ecosystems. Moreover, these comments are in line with Boomsma \& Steg's research (2014), on the abilities of individuals who are aware of environmental considerations to accept reduced lighting levels. Within the two studies, the solution of mobile sensors is appreciated for their ability to automatize the variation of intensity and, according to one comment, to give "the impression of being welcomed by the light!". Beyond the detection of moving objects, the other integrated sensors did not succeed in verifying the interest of this innovation to the community. Indeed, the data on air quality, sound, temperature and hygrometry levels have not yet found a way to be used by public services. One element, currently under study, could be of interest: the measure of the reduction in energy consumption of one lamp with intensity variation. As it is factual, the measure cannot be a precise estimate on a big scale, as the variety of lighting material and associated electricity consumption require deeper and more targeted studies, in particular in terms of nocturnal uses of one space - a space that is little visited at night could claim more significant energy savings than a frequently visited site, the increase in light flux is different depending on the case. Lastly, the sensory indicators collected during the studies provide results in terms of the relation between light level and feeling of comfort: the evaluators mainly consider that the variation in intensity was not bothering them - except when it was too brutal - and that the proposed light levels, sometimes lower than normative indications, ensured a pleasant ambiance in the street. 


\section{Discussion}

The results from these field studies allowed to collect factual elements on the perception of public lighting with a variation in intensity, in real conditions. The implementation of physical and digital survey tools, to be completed autonomously by the evaluators, was appreciated, and the different lighting speed parameters allowed to identify sources of discomfort for the evaluators (glare from the harsh light flux increase). The results support the citizens' views as relevant and the important of technological innovation for the communities - in particular about energy savings and light nuisance reduction - and the study of the connections between comfort and lighting level. However, they must be modulated by two elements: the scale of the studied site, i.e. rue La Noue Bras de Fer in Nantes, and the evaluators' familiarity with their environment, especially those of the second study, recruited as residents of the site. Indeed, the potential reproduction of this type of evaluation on a bigger scale (neighbourhood, city, metropolis) can raise questions on the varieties of public spaces. Could a lighting device with intensity variations and the same dimming parameters create the same perceptions in various environments? The scope of the evaluators' knowledge of the place should also be taken into consideration: would a casual walker give the same answers as a resident? Lastly, the results from these citizen consultations could become elements to support renovation policies in public lighting, which are led by an increasing number of French and international municipalities and metropolises since the arrival of LED systems on the lighting market. In that sense, we can question the desire of cities and metropoles to rely on citizen consultation tools to accompany their actions for the renewal of the lighting stock.

\section{References}

1. W. Schivelbusch, La nuit désenchantée, Éditions Le Promeneur (1993)

2. A. Bovet-Pavy, Lumières sur la ville: Une histoire de l'éclairage urbain, Coéditions François Bourin (2018)

3. S. Challéat, "Sauver la nuit ": empreinte lumineuse, urbanisme et gouvernance des territoires, sous la direction de A. Larceneux, 281-295 (2010)

4. S. Bertin, S. Paquette, Apprendre à regarder dans l'obscurité : les « entre-deux " du paysage urbain nocturne, Environnement urbain /Urban Environment, 9, 1-20 (2017)

5. S. Mosser, Éclairage et sécurité en ville : l'état des savoirs, Déviance et Société, 31, 77-100 (2007)

6. A. Peña-Garcia, A. Hurtado, M. Aguilar-Luzón, Considerations about the impact of public lighting on pedestrians' perception of safety and well-being, Safety Science, 89, 315-318 (2016)

7. J.M. Deleuil, Evalum : de l'évaluation de différentes sources lumineuses par les usagers, in J.M. Deleuil (dir.), Éclairer la ville autrement, Innovations et expérimentations en éclairage public, Presses polytechniques et universitaires romandes, 37-52 (2009)

8. J.M. Deleuil, De l'acceptabilité sociale des diminutions d'éclairement : une expérimentation lyonnaise, in J.M. Deleuil (dir.), Éclairer la ville autrement, Innovations et expérimentations en éclairage public, Presses polytechniques et universitaires romandes, 55-67 (2009) 
9. M. Fontoynont, J.M. Deleuil, Sodium haute pression vs iodures métalliques comparaison des performances visuelles sur un site urbain, in J.M. Deleuil (dir.), Éclairer la ville autrement, Innovations et expérimentations en éclairage public, Presses polytechniques et universitaires romandes, 70-83 (2009)

10. C. Boomsma, L. Steg, The effect of information and values on acceptability of reduced street lighting, Journal of Environnemental Psychology, 39, 22-31 (2014)

11. C. Boomsma, L. Steg, Feeling safe in the dark: examining the effect of entrapment, lighting levels, and gender on feelings of safety and lighting policy acceptability, Environment and Behavior, 46, 193-212 (2014)

12. T. Edensor, Reconnecting with darkness : gloomy landscapes, lightless places, Social \& Cultural Geography, 14, 446-465 (2013)

13. T. Edensor, Rethninking the relationship between light and dark, Urban Studies, 52, 422-438 (2015)

14. T. Edensor, M. Cook, Cycling through dark space : apprehending landscape otherwise, Mobilities, 12, 1-19 (2017),C. Boomsma, L. Steg, The effect of information and values on acceptability of reduced street lighting, Journal of Environmental Psychology, 39, 22-31 (2014) 